\section{Progress in cardiology}

Edited by Paul N. Yu and John F. Goodwin. Vol 16. Pp 163, with illus. Lea \& Febiger, 600 Washington Sq, Philadelphia, 9106-4198, $1988 \$ 28.50$.

This volume marks the end of Drs. Yu and Goodwins' active editorship that began with the first volume in 1972, entitled Computers in cardiology. Since then, these volumes have witnessed the maturation of coronary artery surgery, percutaneous coronary angioplasty, and thrombolysis. Likewise, enormous progress has occurred in echocardiography as well as the increasingly sophisticated nuclear cardiology.

Current contributors come primarily from the University of Pittsburgh School of Medicine, Belfast, and London. Jane Somerville, MD, of the $\mathrm{Na}$ tional Heart Hospital, London, presents "Heart disease in the adolescent," which includes surgical problems in the cyanotic and acyanotic patients. Medical problems associated with cardiac rhythm disturbances, myocardial dysfunction, endocarditis, and pulmonary hypertension are discussed. Problems generally associated with adolescencedriving, insurance coverage, psychosocial difficulties - are surveyed as well. Because most of us have little contact with teens, these guidelines prove quite useful.

Lipid transfer disorders, including etiology, diagnosis, and treatment, are thoroughly reviewed by Antonio M. Gotto, Jr, MD, of the Baylor College of Medicine, Houston, Texas.

Those not involved in the rather esoteric endeavor of heart and lung transplantation will find the chapter detailing this subject interesting. Occasionally patients ask about organ transplant procedures; at least we should be able to speak somewhat assuredly about this limited area.

Cardiologist D. Boyle, MD, at the
Royal Victoria Hospital in Belfast comprehensively details present-day approaches to acute myocardial infarction. The section on thrombolytic therapy confirms the crucial role that timing plays in assessing this procedure's efficacy, while illustrating the futility of delayed intervention. Including the section covering the available pharmacologic agents used to prevent acute myocardial infarction is especially timely.

Ronald F.W. Campbell, MD, from the University of Newcastle-uponTyne, offers an excellent discussion concerning antiarrhythmic agents. He categorizes each drug action according to the Vaughan Williams classification and reviews therapeutic drug monitoring.

A discussion of hypertension closes this volume, specifically childhood assessment and a survey of trials in hypertension. The latter pays particular attention to the drug treatment results in uncomplicated, mild hypertension; the relationship between high blood pressure and coronary disease and cardiovascular complications; and the role of beta blockers and thiazide diuretics.

This edition continues the quality standards established by the outgoing editors, and I am confident subsequent volumes will adhere to this tradition.

\section{CARSON TODD, DO, FACOI \\ Tulsa, Okla}

\section{Life-threatening dermatoses}

By Paul A. Krusinki, and Franklin P. Flowers. Pp 300, with illus. 35 E Wacker Dr, Chicago, 1987, \$53.50.

Many physicians do not view dermatology as a medical specialty that routinely deals with serious or life-threatening diseases. In fact, skin manifestations of systemic illnesses are relatively common and will increase in numbers as immunodeficiency disorders and advances in immunotherapy become more prominent. Additionally, because many dermatologic disorders are associated with significant morbidity and mortality, especially when not diagnosed early, correct diagnosis and appropriate treatment are mandatory. To that end, Life-threatening dermatoses presents a complete, yet concise, review of "imperative diagnoses that should not be missed." In a single source, this text compiles the various approaches to clinical diagnosis and management of potentially serious skin disorders. While similar information can be found scattered throughout the medical literature, no one text has attempted this comprehensive task.

Each chapter reviews individual diseases and helps the reader differentiate conditions by clinical and laboratory means. Diffuse erythemas, collagen vascular diseases, opportunistic infections and AIDS, malignant melanoma and its precursor lesions, bullous diseases, and life-threatening diseases in pediatrics, (skin manifestations indicating child abuse) represent some of the topics discussed.

The text's main attributes can be found in the clinical evaluation, logical ordering and interpretation of laboratory data, and diagnostic suggestions for commonly encountered situations. Immediate frozen section skin biopsy for use in the early diagnosis of toxic epidermal necrolysis, and appropriate skin biopsy technique of lesions suspicious for malignant melanoma illustrate the latter. Reviewed succinctly, yet accurately, treatment encompasses all the important concepts.

Although written by different authors, the book features consistent style and design. The editors deserve special recognition for this feat. Well referenced chapters display helpful 


\section{This month,}

8 million Americans

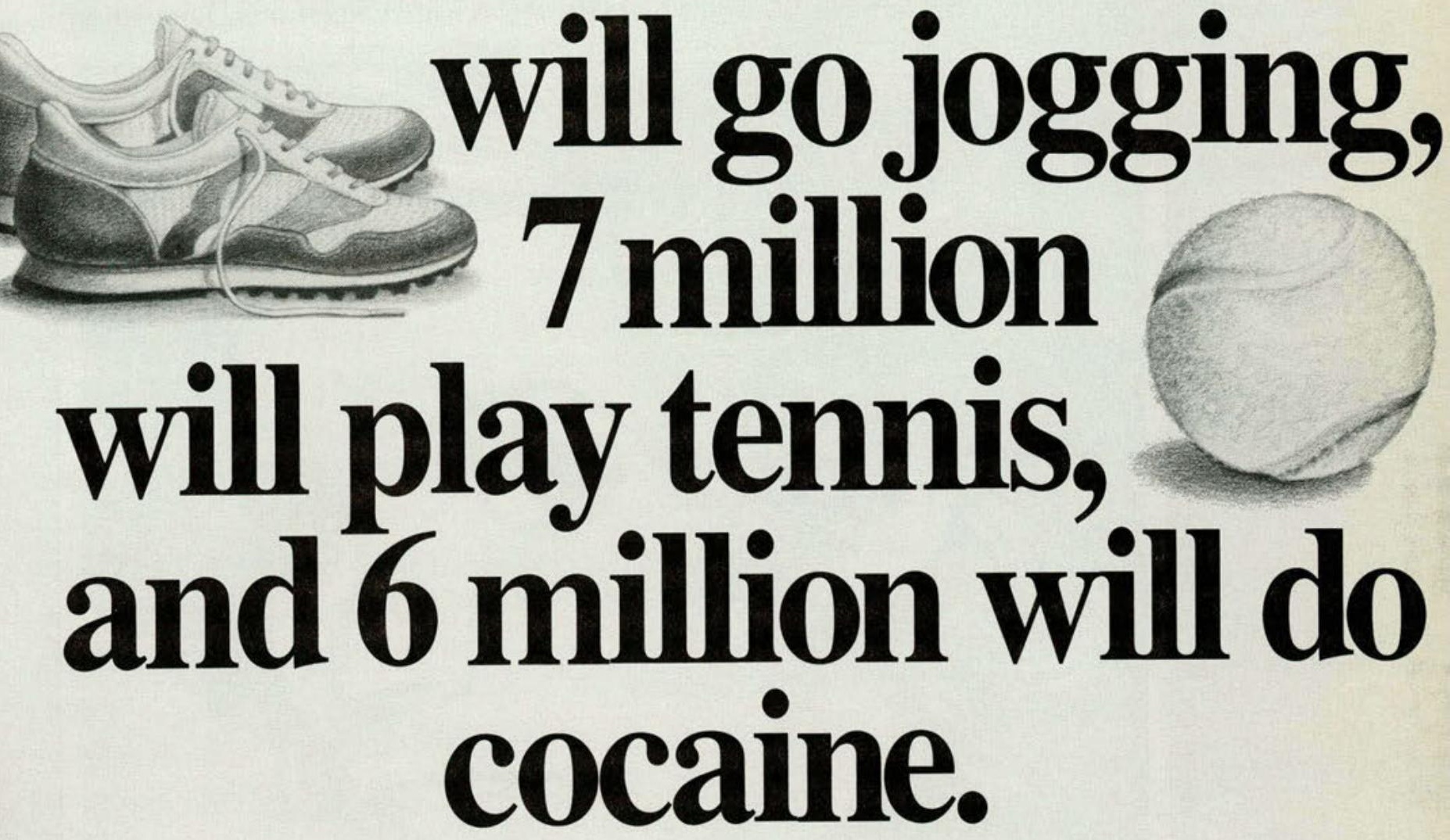

A few of those

6 million may be

your patients.

You may have trouble

recognizing cocaine

abuse because there are few

visible symptoms. Often the only way

to find out is to ask the right questions.

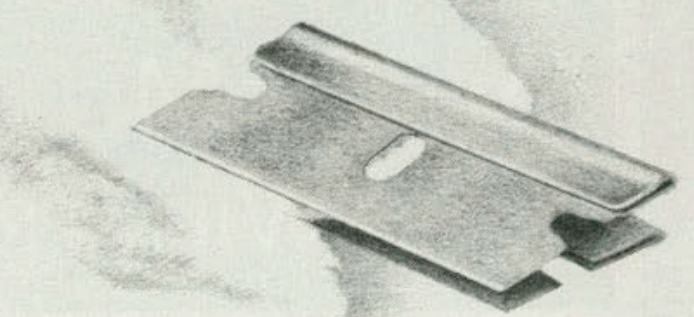

Any cocaine use is

potential abuse. Give your patients who have a problem the 1-800-COCAINE hotline or the National Institute on Drug Abuse information and referral line (1-800-662-HELP). 


\section{Together We Can Make A Difference}

Recognizing the need to market their practices locally, many DOs have asked for a professional black-and-white ad that can be used in their community newspapers. Now you can have either or both of the ads on these pages free if you are an AOA member or affiliate, or for $\$ 25$ each if you aren't. We'll provide the ads, ready to have your name and address inserted in the box at the bottom; you pay for the space. Show the sample to your paper, get the space charges, then request camera ready copies from the AOA Public Relations Department.

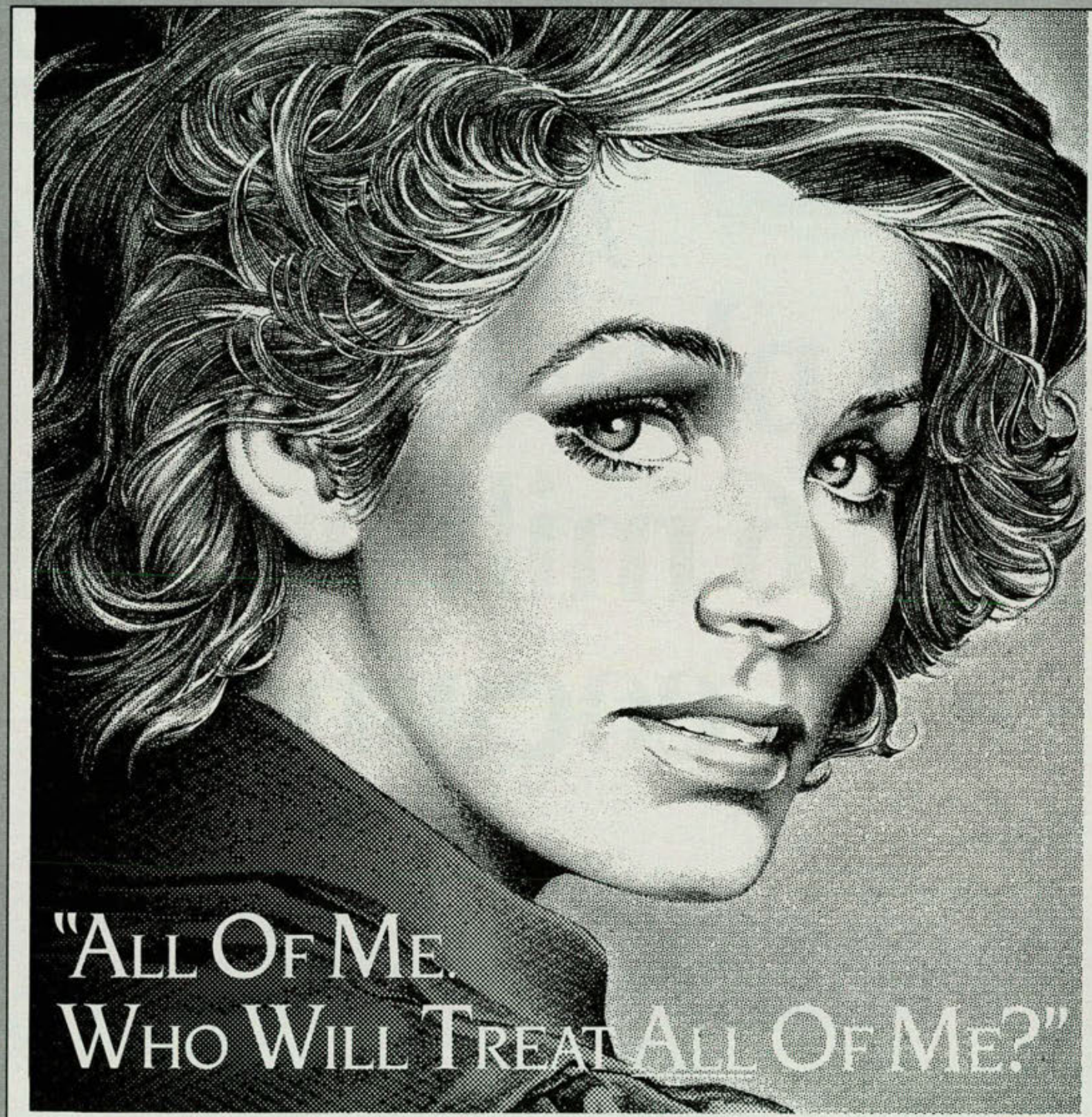

You sometimes wonder-we know you do - whether any physician has ever viewed you as a whole person, with needs that extend beyond the part of you that hurts. Emotional. Nutritional. Physiological needs. One specific type of doctor, the Osteopathic Physician, is qualified to care for all of you. For every aspect of you as a personyour physical well-being, emotional health, your nutritional needs, and those of every member of your family.

It's reassuring to know that in this world of fragmented medical care, someone still cares for all of you. 


\section{THERE IS}

A DOCTOR

FOR ALL OF

\section{YOU.}

\section{BY EDUCATION,} TRAINING, AND CHOICE.

One specific type of physician is qualified to care for all of you. To care for every aspect of you as a person-your physical well-being, emotional health, your nutritional needs, and those of every member of our family.

An Osteopathic Physician is a family oriented doctor-a general practitioner or other specialist-someone who recognizes you as a person with needs that extend beyond the part of you that hurts on any given day.

It's good to know that in this world of fragmented medical care, someone still: cares for all of you.
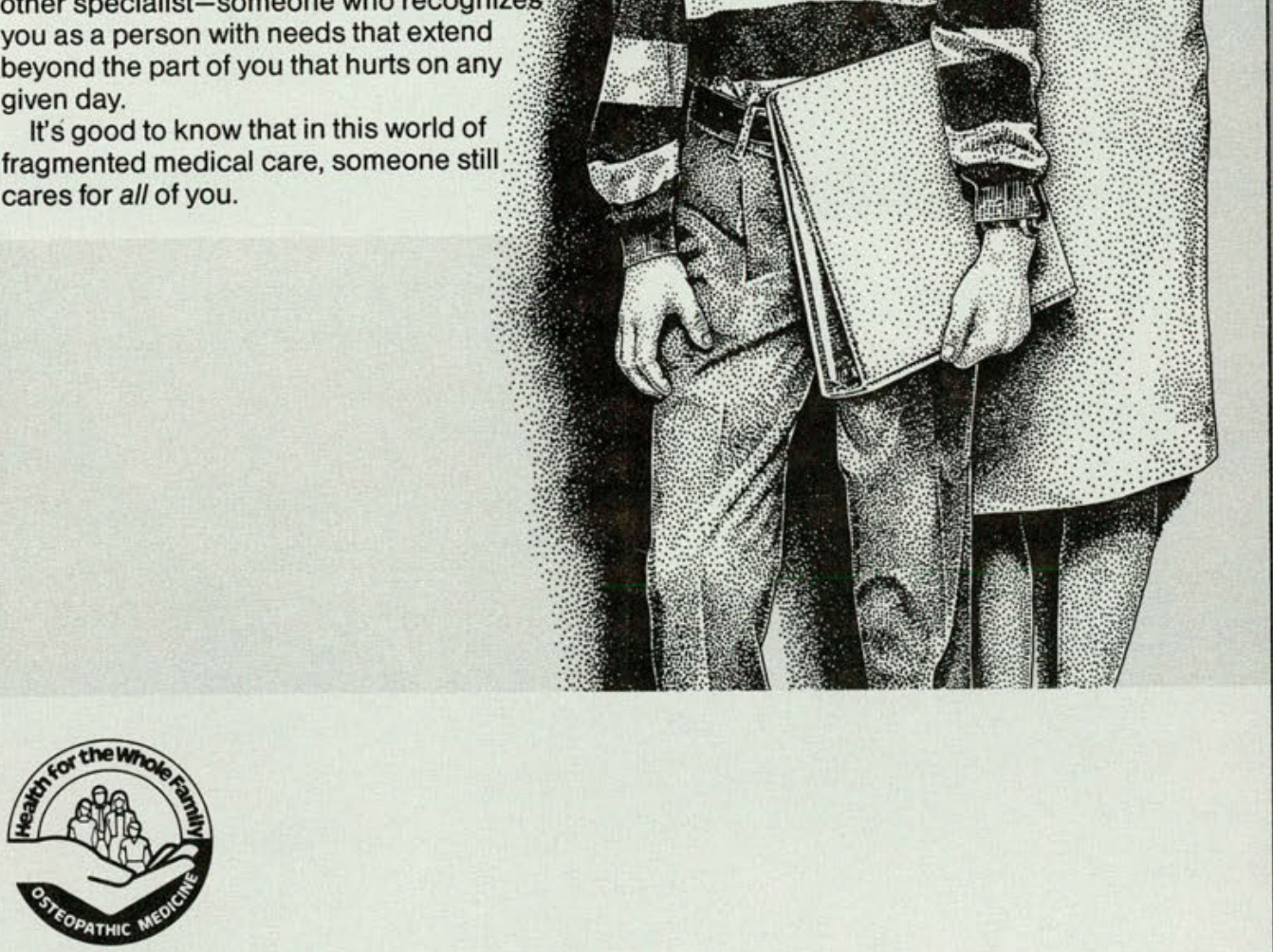

\section{Together We Are Making A Difference}

The ads are offered courtesy of the Auxiliary to the American Osteopathic Association and the AOA, free or at nominal cost. They are part of the national ad campaign initiated by the AAOA, which will also include some color ads in national magazines. The costs are covered by contributions to the Auxiliary, so if you would like to help it to continue the program, please send your contribution to the National Ad Campaign, Auxiliary to the AOA, 142 E. Ontario St., Chicago, IL 60611. 

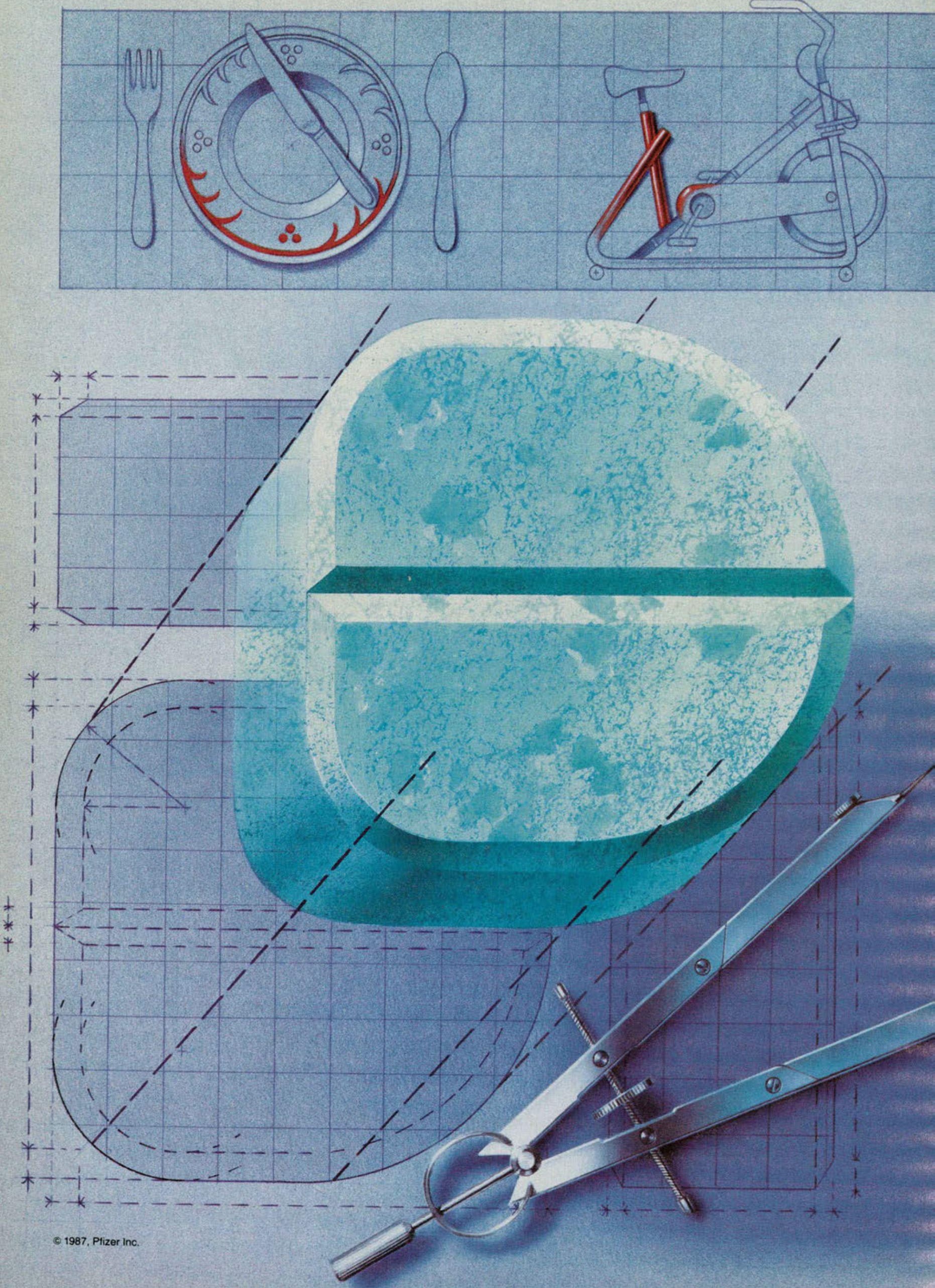

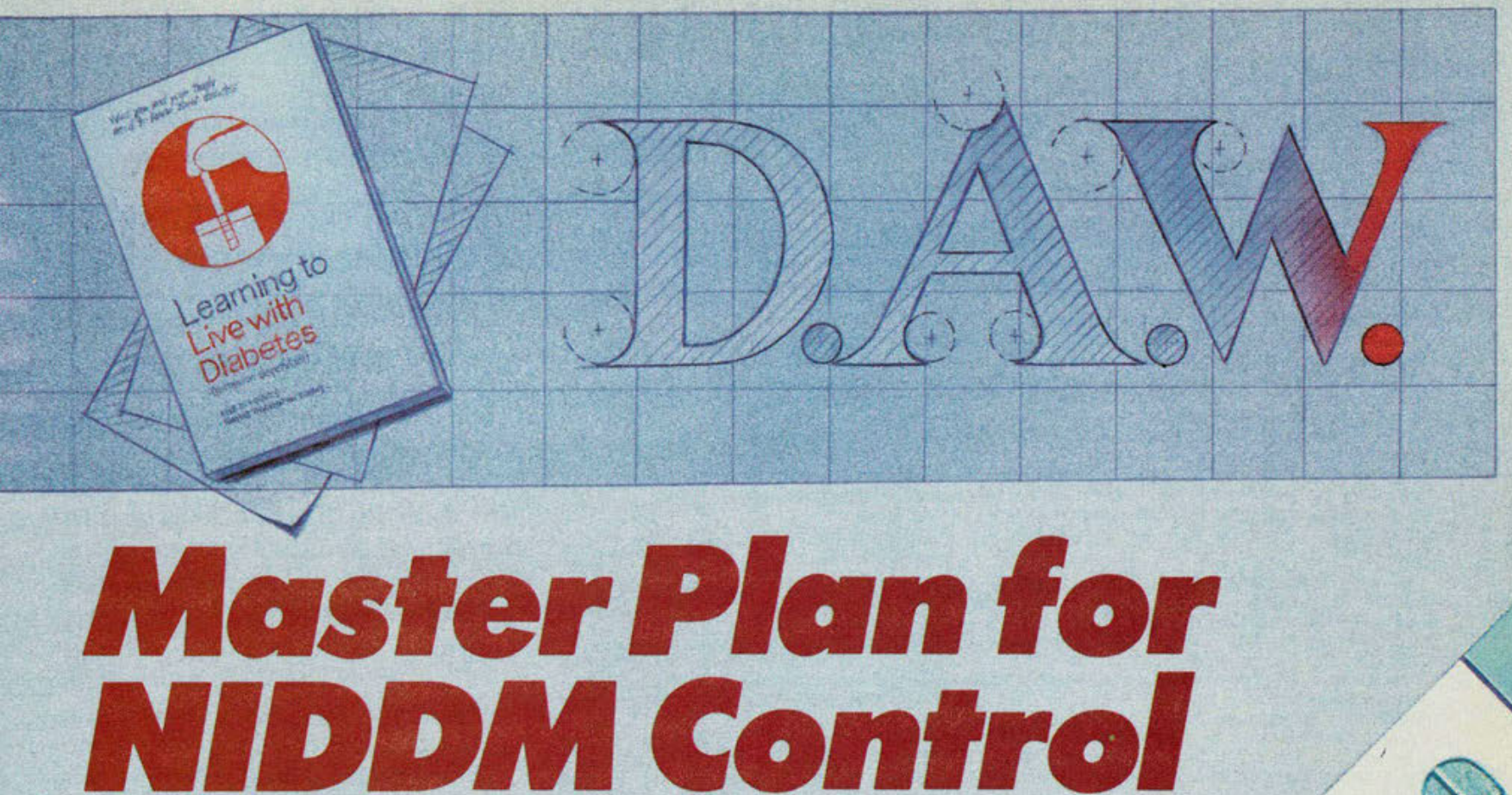

\section{A Diabinese ${ }^{\circledR}$ (chlorpropamide) prescription completes the design}

Diet, exercise, education and once-daily Diabinese, dispensed as written, comprise the Master Plan for successful non-insulin-dependent diabetes mellitus (NIDDM) control. For overall NIDDM control, specify Diabinese ${ }^{\circledR}$ by name.

\section{Proven Efficacy and Safety}

In a two-year study comparing Diabinese to glyburide, "...chlorpropamide was clinically more effective with a smaller number of primary and secondary drug failures and a greater proportion of patients successfully controlled at the end of 2 years. Severe hypoglycemia was

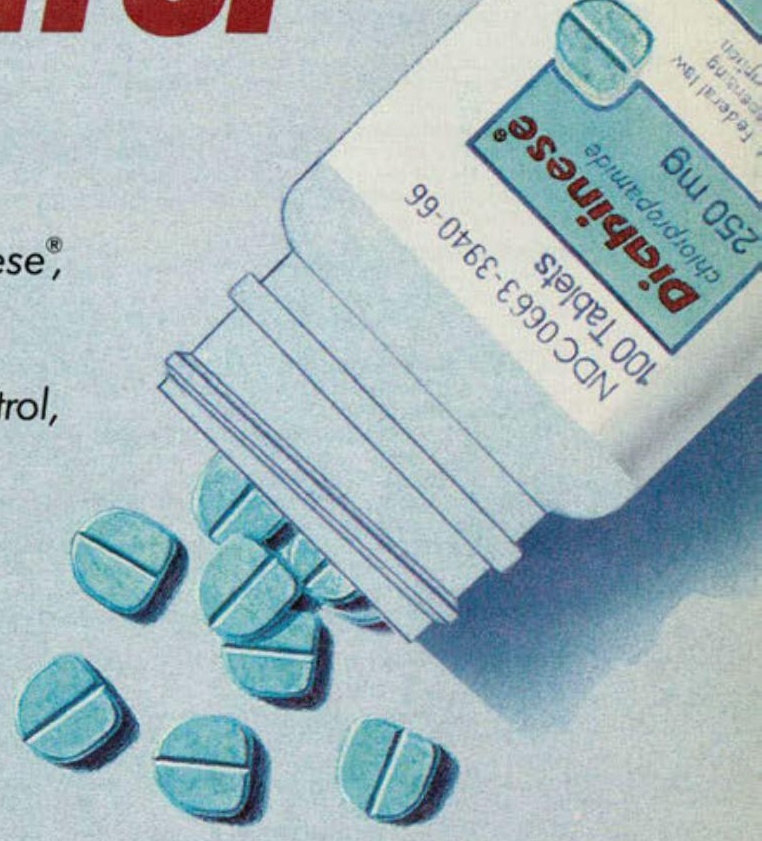
a greater hazard during treatment with glyburide...."

As with all sulfonylureas, hypoglycemia may occur with Diabinese.

\section{Once-a-day}

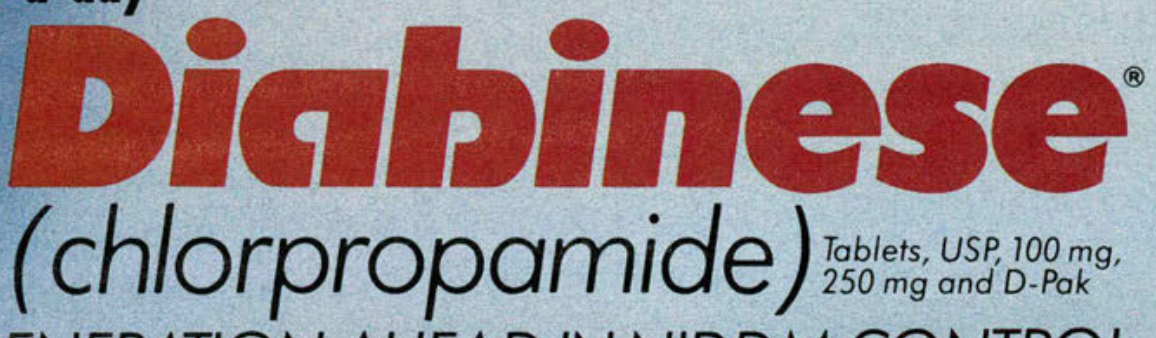
A GENERATION AHEAD IN NIDDM CONTROL Reference: 1. Clarke BF: Comparative effectiveness of glyburide in the treatment of non-insulin-dependent diabetes, in
Diagnosis and Management of Diabetes Mellitus. Postgraduate Medicine: Custom Communications, April 1982, pp 57-65. 


\section{SPECIAL WARNING ON INCREASED RISK OF CARDIOVASCULAR MORTALITY}

The administration of oral hypoglycemic drugs has been reported to be associated with increase cardiovascular mortality as compared to treatment with diet alone or diet plus insulin. This warnin is based on the study conducted by the University Group Diabetes Program (UGDP), a long-term prospective clinical trial designed to evaluate the effectiveness of glucose-lowering drugs in preventing or delaying vascular complications in patients with non-insulin-dependent diabetes. The study involved 823 patients who were randomly assigned to one of four treatment groups (Diabetes, 19 (supp. 2]:747-830, 1970).

treated for 5 to 8 years with diet plus a fixed dose of tolbutamide (1.5 UGDP reported that patients treated tor 5 to grams per day) had a rate of cardiovascular mortality approximately $21 / 2$ times that of patients treated with diet alone. A significant increase in total mortality was not observed, but the use of tolbutamide was discontinued based on the increase in cardiovascular mortality, thus limiting the opportunity for the study to show an increase in overall mortality. Despite controversy regarding the interpretation of these results, the findings of the UGDP study provide an adequate basis for this warning. The patient should be informed of the potential risks and advantages of DIABINESE (chlorpropamide) and of alternative modes of therapy.

Although only one drug in the sulfonylurea class (tolbutamide) was included in this study it is prudent from a safety standpoint to consider that this warning may also apply to other oral hypoglycemic drugs in this class, in view of their close similarities in mode of action and chemical structure.

PRECAUTIONS

General

Hypoglycemia: All sulfonylurea drugs are capable of producing severe hypoglycemia. Proper patient selection, dosage, and instructions are important to avoid hypoglycemic episodes. Renal or hepatic insufficiency may cause elevated blood levels of DIABINESE and the latter may also diminish gluconeogenic capacity both of which increase the risk of serious hypoglycemic reactions. Elderly debilitated or malnourished patients, and those with adrenal or pituitary insufficiency are particularly susceptible to the hypoglycemic action of glucose-lowering drugs. Hypoglycemia may be difficult t recognize in the elderly, and in people who are taking beta-adrenergic blocking drugs. Hypoglycemia is more likely to occur when caloric intake is deficient, after severe or prolonged exercise, when alcohol is

ingested, or when more than one glucose-lowering drug is used.

Because of the long half-life of chlorpropamide, patients who become hypoglycemic during therapy require careful supervision of the dose and frequent feedings for at least 3 to 5 days. Hospitalization and intravenous glucose may be necessary.

Loss of control of blood glucose. When a patient stabilized on any diabetic regimen is exposed to stress such as fever trauma, infection, or surgery, a loss of control may occur. At such times, it may be necessary to discontinue DIABINESE (chlorpropamide) and administer insulin.

The effectiveness of any oral hypoglycemic drug. including DIABINESE, in lowering blood glucose to a desired level decreases in many patients over a period of time, which may be due to progression of the severity of the diabetes or to diminished responsiveness to the drug. This phenomenon is known a secondary failure, to distinguish it from primary failure in which the drug is ineffective in an individua patient when first given.

AOVERSE REACTIONS

Hypoglycemia: See PRECAUTIONS section

Gastrointestinal Reactions: Cholestatic jaundice may occur rarely: DIABINESE should be discontinue if this occurs. Gastrointestinal disturbances are the most common reactions: nausea has been reported in less than $5 \%$ of patients, and diarrhea, vomiting, anorexia, and hunger in less than $2 \%$. Other gastrointestinal disturbances have occurred in less than $1 \%$ of patients including proctocolitis. They ten to be dose related and may disappear when dosage is reduced.

Dermatologic Reactions: Pruritus has been reported in less than $3 \%$ of patients. Other allergic skin reactions, e 8 . urticaria and maculopapular eruptions have been reported in approximately $1 \%$ or less patients. These may be transient and may disappear despite continued use of DIABINESE (chlorpropa patients. These may be transient and may disappear despite continu

Porphyria cutanea tarda and photosensitivity reactions have been reported with sulfonylureas.

Skin eruptions rarely progressing to erythema multiforme and exfoliative dermatitis have also been reported Hematologic Reactions: Leukopenia, agranulocytosis, thrombocytopenia, hemolytic anemia, aplastic anemia, pancytopenia, and eosinophilia have been reported with sulfonylureas.

Metabolic Reactions: Hepatic porphyria and disulfiram-like reactions have been reported with DIABINESE Endocrine Reactions: On rare occasions, chlorpropamide has caused a reaction identical to the syndrome of inappropriate antidiuretic hormone $(\mathrm{ADH})$ secretion. The features of this syndrome result from excessive water retention and include hyponatremia, low serum osmolality and high unine osmolality.

\section{DOSAGE AND ADMINISTRATION}

There is no fixed dosage regimen for the management of diabetes mellitus with DIABINESE or any other ypoglycemic agent in addition to the usual monitoring of urinary glucose, the patient's blood glucose must also be monitored periodically to determine the minimum effective dose for the patient: to detect primary failure, and to detect secondary failure. Glycosylated hemoglobin levels may also be of value in monitoring the patient's response to therapy

The total daily dosage is generally taken at a single time each morning with breakfast. Occasionally cases of gastrointestinal intolerance may be relieved by dividing the daily dosage. A LOADING OR PRIMING COSES IS NOT NECESSARY AND SHOULD NOT BE USED.

Initial Therapy: 1 . The mild to moderately severe, middle-aged, stable, non-insulin-dependent diabetic patient should be started on $250 \mathrm{mg}$ daily. Older patients should be started on smaller amounts of DIABINESE (chlorpropamide), in the range of 100 to $125 \mathrm{mg}$ dail,

2. No transition period is necessary when transferring patients from other oral hypoglycemic agents to DIABINESE. The other agent may be discontinued abruptly and chlorpropamide started at once. In prescribing chlorpropamide, due consideration must be given to its greater potency.

Many mild to moderately severe, middle-aged stable non-insulin-dependent diabetic patients receiving insulin can be placed directly on the oral drug and their insulin abruptly discontinued. For patients requiring more than 40 units of insulin daily therapy with DIABINESE may be initiated with upon the response.

Five to seven days after the initial therapx the blood level of chlorpropamide reaches a plateau. Dosage may subsequently be adjusted upward or downward by increments of not more than 50 to $125 \mathrm{mg}$ at intervals of 3 to 5 days to obtain optimal control. More frequent adjustments are usually undesirable.

Maintenance Therapy: Most moderately severe, middle-aged. stable non-insulin-dependent diabetic patients are controlled by approximately $250 \mathrm{mg}$ daily. Many investigators have found that some milder diabetics do well on daily doses of $100 \mathrm{mg}$ or less. Many of the more severe diabetics may require $500 \mathrm{mg}$ daily for adequate control. PATIENTS WHO DO NOT RESPOND COMPLETELY TO 500 MG DAIIY WILL USUALLY NOT RESPOND TO HIGHER DOSES. MAINTENANCE DOSES ABOVE 750 MG DAILY SHOULD BE AVOIDED. HOW SUPPLIED

Blue, 'D'-shaped, scored tablets in strengths of $100 \mathrm{mg}$, tablet code 393: (100's. NDC \#0663-3930-66. 500's. NDC \#0663-3930-73; and 100 unit dose of $10 \times 10$. NDC \#0663-3930-41) and $250 \mathrm{mg}$. tablet code 394: (100's. NDC \#0663-3940-66:250's. NDC \#0663-3940-71; 1000's. NDC \#0663-3940-82:100 unit dose of $10 \times 10$, NDC \#0663-3940-41; and 30's D-Pak. NDC \#0663-3940-301

RECOMMENDED STORAGE: Store below $86^{\circ} \mathrm{F}\left(30^{\circ} \mathrm{C}\right)$.

CAUTION: Federal law prohibits dispensing without prescription charts. These concentrate on significant differential diagnoses, common cutaneous findings, and important disease associations. Likewise, quality paper, easy-to-read print, and a hard cover enhance the book's quality.

Algorithms follow each chapter, another potential advantage. Each one guides the reader through a specific clinical scenario-anaphylaxis, diffuse macular erythema, purpura, and the like-in an attempt to consider the most significant disorders in the differential diagnosis. The editors preface the use of algorithms as primarily clinical exercises, noting that they do not ensure correct diagnosis nor replace good clinical judgement. With that in mind, the algorithms presented here prove valuable in working through clinical situations and can help the physician avoid missing a disease with serious implications. Algorithms and clinical decision making are specifically addressed in the last chapter. More complex and different in scope than the previous chapters, this well written section contains material extremely important to all physicians making patient management decisions.

Incorporating actual case studies would have enhanced not only the proper use of algorithms, but clinical and laboratory diagnosis and biopsy technique and interpretation as well.

While text and charts are admirable, the black and white photographs offer little clinical value. They seemed chosen at random. In most cases, color photographs are required to capture the clinical morphology. However, small color plates can be found in the middle section of the book. They represent reasonably good quality for such a text.

Nonetheless, including color photographs of disease entities and bedside laboratory test (Tzanck smear, potassium hydroxide prep) would prove most effective.

Life-threatening dermatoses is best viewed as an excellent introductory text or reference that fills an important void among medical textbooks, rather than as a complete general dermatology text. Any physician who wishes to develop a sound 


\section{CERTIFICATION OF HOME STUDY}

This is to certify that I,

activity for AOA CME credits.

Please print

completed the following

Type of activity (such as reading or listening)

Vame of journal(s) or audio-tape and date(s) of issue(s):

(One-half CREDIT may be granted for each issue or tape)

The Home Study form is intended to document individual reading of recognized scientific journals, listening to approved audio-tapes, and other approved home study courses and programs under the criteria described for Category 2-B.

Only one type of home study, such as reading, should be indicated on a single form, though multiple issues of scientific journals may be listed.

This form should not be used, however, when CME quiz cards for the AOA Journal are submitted separately.

\begin{tabular}{|l}
\hline FOR OFFICE USE ONLY \\
\hline Date \\
Program \# Credits \\
Doctor \# \\
Doctor's Name
\end{tabular}


diagnostic and management approach to common and uncommon, potentially life-threatening skin diseases can benefit from this book. It should be on the shelf of all teaching hospital libraries, and on the reading list of all second-year dermatology residents. Internal medicine and family practice residents also will benefit from reading this text.

I hope the editors will provide frequent editions to keep up with rapid advances in diagnosis and therapy.

JAMES Q. DEL ROSSO, DO

Athens, Ohio

\section{Handbook of drug therapy in psychiatry}

By Jerrold G. Bernstein. Ed 2. Pp 586, with illus. PSG Publishing $\mathrm{Co}$, Inc, 545 Great Rd, PO Box 6, Littleton, MA 01460, $1988, \$ 39.50$.

Today, psychotropic medications are among the most common drugs used by practicing physicians, irrespective of their specialty. Unfortunately, nonpsychiatrists receive much of their information about them primarily from drug companies. While a thorough, comprehensive understanding of psychotropic drug use could previously only be acquired by reviewing articles published by clinicians and researchers, this book offers another alternative. As a psychiatrist, I have read various texts on the subject over the years. However, I have never come across a more comprehensive overview, presented in such an interesting, informative fashion.

Written after the publication of the Diagnostic and statistical manual of mental disorders (DSM-III R), this edition covers each of the major classifications of mental disorders, including drugs employed. Each chapter begins with a concise overview of the main points made within. I found this extremely helpful, both as a preview of the major topics to be discussed, and as a summary thereafter.

Many biological aspects of psychiatric illnesses and action mechanisms of psychotropic medications are detailed in a very concise, interesting, informative, and practical manner. Nonpsychotropic drugs- uses and side effects-have been included as well, along with recreational drug use and addiction.

As a general psychiatrist who devotes a fair amount of time to consultation and liaison psychiatry in a general hospital, I am often asked by my colleagues to share my expertise on psychotropic medications. I will, undoubtedly, recommend Handbook of drug therapy in psychiatry to them. It represents the single best book I have ever read on this topic. In my opinion, it will make an excellent addition to any physician's bookshelf.

\section{J. BARRY RUBIN, DO \\ Madison Heights, Mich}

\section{Kaposi's sarcoma: A text and atlas}

Edited by Geoffrey J. Gottlieb, and A. Bernard Ackerman. Pp 330, with illus. Lea \& Febiger, 600 Washington Sq, Philadelphia, 19106-4198, 1988, \$98.50.

This book boasts an impressive list of specialists as contributors. Certainly not meant for casual reading, the highly technical text demands detailed study.

Excellentbackground information provides an interesting history of the disease's namesake as well as the sarcoma itself. Extensive bibliographies are included for those who desire more information or care to pursue the original writings.

Perhaps most important is the discussion of Kaposi's sarcoma as it relates to the acquired immune deficiency syndrome (AIDS). Dr. Kaposi (nee Kohn) probably never anticipated the impact this disease would have today.

Photomicrographs of the tissue sections are outstanding. All varieties of the disease are illustrated and accompanied by detailed descriptions. Likewise, the color photos offer exceptional clarity and completeness. The differential diagnosis is superbly presented in both text and comparative photographs.

Systemic manifestations and immunopathologies receive interesting coverage in separate chapters.

Kaposi's sarcoma: A text and atlas is a must for dermatopathologists, dermatologists, and infectious disease specialists. The general pathologist and those involved in public health may find it a useful adjunct, especially the former who perform post mortem examinations of AIDS patients, and the latter for their interest in epidemiology.

The book's preface states a need for comprehensive coverage exists. That void is now filled by this text.

HARRY B. ELMETS, DO, FAOCD

Clinical Professor of Dermatology University of Osteopathic Medicine and Health Sciences Des Moines, Iowa

\section{The illness narratives: Suffering, healing, and the human condition}

By Arthur Kleinman. Pp 284. Basic Books, Inc, 10 E 53rd St, New York, $10022,1988, \$ 19.95$.

As Richard Selzer wrote in an article in the Aug 21, 1988, New York Times Magazine,"A doctor walks in and out of a dozen short stories a day. It is irresistible to write them down."

One such note-taker is Dr. Arthur Kleinman, a psychiatrist and professor of anthropology at Harvard Medical School. Dr. Kleinman writes, "Each patient brings to the practitioner a story. That story enmeshes the disease in a web of meanings that make sense only in the context of a particular life. But to understand that life and the illness experience it creates, we must relate life and illness to cultural context."

One of the major drawbacks of Dr. Kleinman's book is that academic opacity and formal description too often encumber an otherwise clear, concise work.

Immediately following this wonderful discussion on patient interpretation quoted above, Dr. Kleinman notes, "American women of the middle class who articulate somatic idioms of distress communicate their distinctive biological and psychological problems together with the shared cultural tension of an unresolved conflict between the traditional expectation of family life and the desire, intensified by contemporary social pressures, to experience personal freedom." Not even a top-notch professor can say that in one breath, let 
A good choice

is at your fingertips...
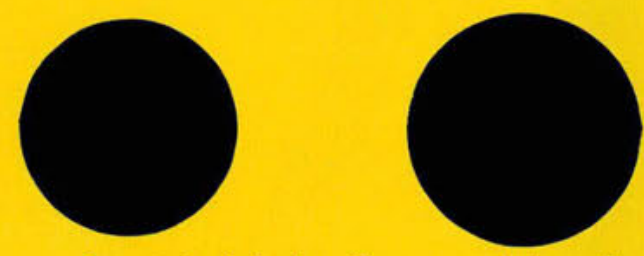

Insert left index finger and thumb, and turn the page. 


\section{for many osteoarthritic patients age 65 and older}

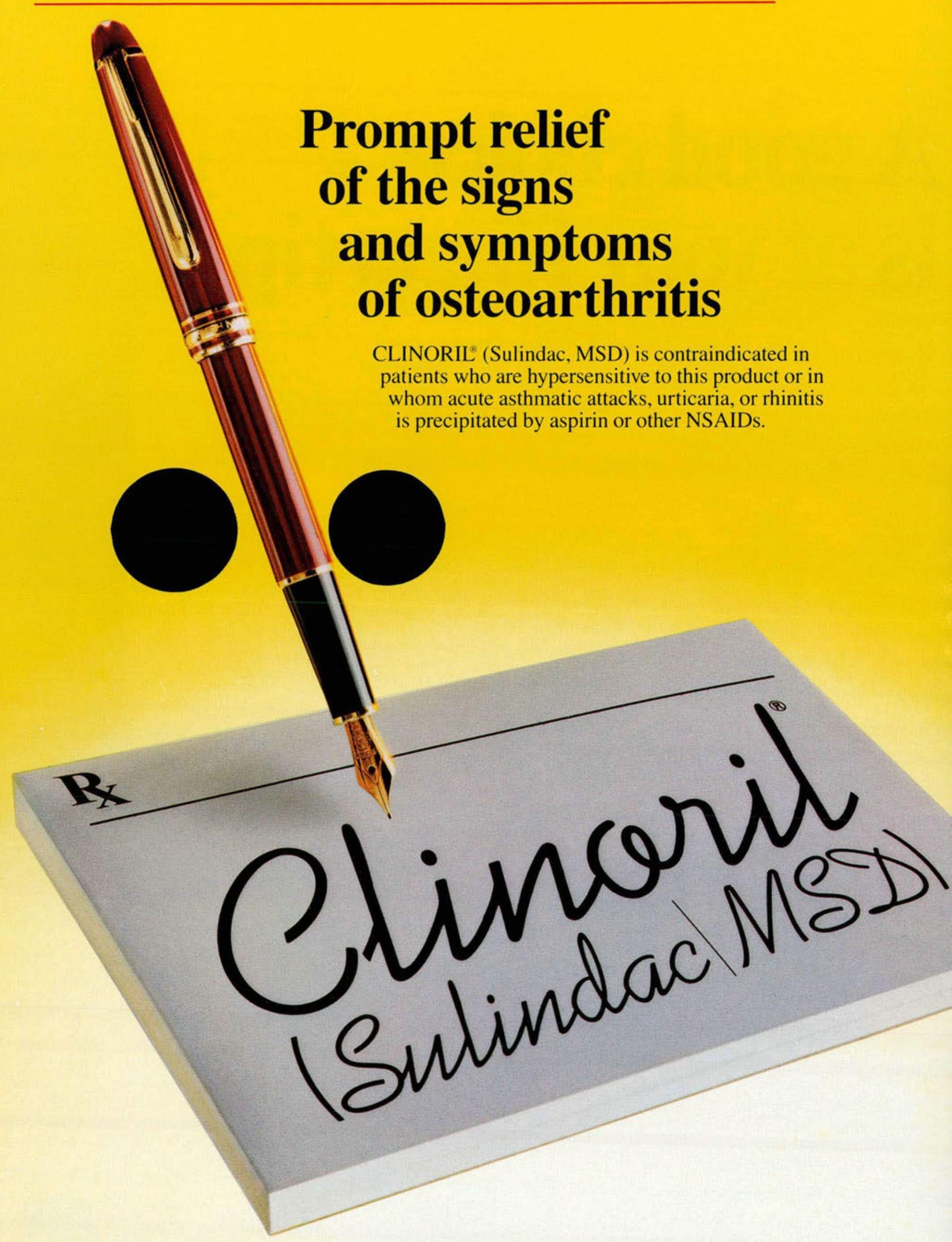




\section{CLINORIL may affect} renal function to a lesser extent than other NSAIDs; however, renal adverse effects have been reported

Caution should be exercised when administering CLINORIL to patients with conditions associated with increased risk of the effects of NSAIDs on renal function, such as those with complications associated with advanced age.

*These observations need further clarification, and, in the interim, CLINORIL should be used with caution in patients whose renal function may be impaired or prostaglandin dependent. Such patients should be closely monitored.
No reported blunting of certain antihypertensive agents

Edema has been observed in some patients taking CLINORIL. Therefore, as with other NSAIDs, CLINORIL should be used with caution in patients with compromised cardiac function, hypertension, or other conditions predisposing to fluid retention.

For many osteoarthritic patients age 65 and older

Choose
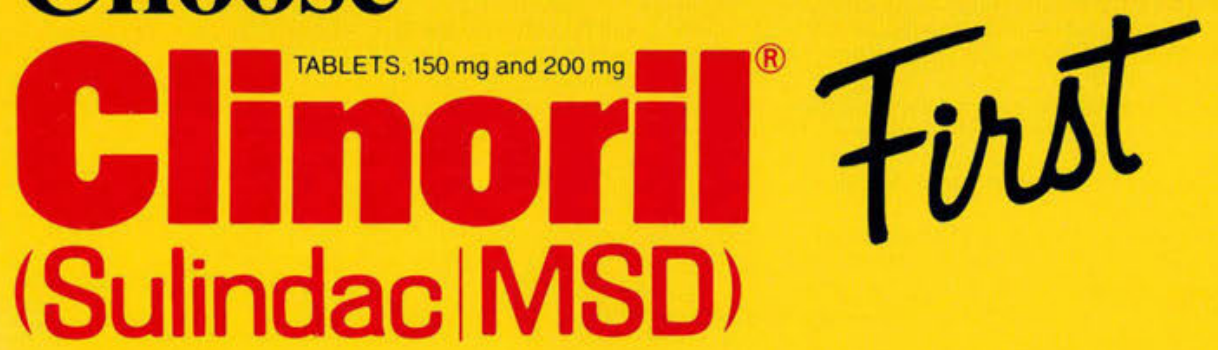
For many osteoarthritic patients age 65 and older Choose (SulindacIMSD) First

Contraindications: Hypersensitivity to this product; patients in whom acute asthmatic attacks, urticaria, or rhinitis is precipitated by aspirin or other nonsteroidal antiinflammatory agents.

Warnings: Peptic ulceration and gastrointestinal bleeding have been reported. Fatalities have occurred. Gastrointestinal bleeding is associated with higher morbidity and mortality in patients acutely ill with other conditions, the elderly, and patients with hemorrhagic disorders. In patients with active gastrointestinal bleeding or an active peptic ulcer, an appropriate ulcer regimen should be instituted, benefits of therapy must be weighed against possible hazards, and the patient's progress carefully monitored; in patients with a history of either upper or lower gastrointestinal tract disease, CLINORIL ${ }^{\circ}$ (Sulindac, MSD) should be given under close supervision and only after consulting the Adverse Reactions section.

Rarely, fever and other evidence of hypersensitivity (see Adverse Reactions), including abnormalities in one or more liver function tests and severe skin reactions, have occurred; fatalities have occurred in these patients. Hepatitis, jaundice, or both, with or without fever, may occur usually within the first one to three months of therapy. Determinations of liver function should be considered whenever unexplained fever, rash or other dermatologic reactions, or constitutional symptoms develop; if unexplained fever or other evidence of hypersensitivity occurs, discontinue CLINORIL. Elevated temperature and abnormalities in liver function caused by CLINORIL characteristically have reverted to normal after discontinuation of therapy. CLINORIL should not be reinstituted in such patients.

In addition to hypersensitivity reactions involving the liver, in some patients the findings are consistent with those of cholestatic hepatitis. As with other nonsteroidal antiinflammatory drugs, borderline elevations of one or more liver tests without any other signs and symptoms may occur in up to $15 \%$ of patients. These abnormalities may progress, may remain essentially unchanged, or may be transient with continued therapy. The SGPT (ALT) test is probably the most sensitive indicator of liver dysfunction. Meaningful ( 3 times the upper limit of normal) elevations of SGPT or SGOT (AST) occurred in controlled clinical trials in less than $1 \%$ of patients. A patient with symptoms and/or signs suggesting liver dysfunction, or in whom an abnormal liver test has occurred, should be evaluated for evidence of the development of more severe hepatic reaction while on therapy with CLINORIL. Although such reactions as described above are rare, if abnormal liver tests persist or worsen, if clinical signs and symptoms consistent with liver disease develop, or if systemic manifestations occur (e.g., eosinophilia, rash, etc.), CLINORIL should be discontinued. In clinical trials, the use of $600 \mathrm{mg} /$ day has been associated with increased incidence of mild liver test abnormalities (maximum recommended dosage is $400 \mathrm{mg}$ per day)

Precautions: General-Although the effect on platelet function and bleeding time is less than with aspirin, CLINORIL is an inhibitor of platelet function; therefore, patients who may be adversely affected should be carefully observed when CLINORIL is administered. Pancreatitis has been reported (see Adverse Reactions); should pancreatitis be suspected, the drug should be discontinued and not restarted, supportive medical therapy instituted, and the patient monitored closely with appropriate laboratory studies (e.g. serum and urine amylase, amylase/creatinine clearance ratio, electrolytes, serum calcium, glucose, lipase, etc.); a search for other causes of pancreatitis as well as those con ditions which mimic pancreatitis should be conducted. Because of reports of adverse eye findings with nonsteroidal anti-inflammatory agents, it is recommended that patients who develop eye complaints during treatment have ophthalmologic studies. In patients with poor liver function, delayed, elevated and prolonged circulating levels of the sulfide and sulfone metabolites may occur. Such patients should be monitored closely; a reduc tion of daily dosage may be required.

Edema has been observed in some patients taking CLINORIL. Therefore, as with other nonsteroidal anti-inflammatory drugs, CLINORIL should be used with caution in patients with compromised cardiac function, hypertension, or other conditions predisposing to fluid retention. CLINORIL may allow a reduction in dosage or the elimination of chronic corticosteroid therapy in some patients with rheumatoid arthritis. However, it is generally necessary to reduce corticosteroids gradually over several months in order to avoid an exacerbation of disease or signs and symptoms of adrenal insufficiency. Abrupt withdrawal of chronic corticosteroid treatment is generally not recommended even when patients have had a serious complication of chronic corticosteroid therapy.

Renal Effects - As with other nonsteroidal anti-inflammatory drugs, long-term adminis tration of sulindac to animals has resulted in renal papillary necrosis and other abnormal renal pathology. In humans, there have been reports of acute interstitial nephritis with hematuria, proteinuria, and occasionally nephrotic syndrome. A second form of renal toxicity has been seen in patients with prerenal and renal conditions leading to a reduction in renal blood flow or blood volume, where renal prostaglandins have a supportive role in the maintenance of renal perfusion. In these patients, the administration of an NSAID may cause a dose-dependent reduction in prostaglandin formation and may precipitate overt renal decompensation. CLINORIL may affect renal function less than other NSAIDs in patients with chronic glomerular renal disease (see CLINICAL PHARMACOLOGY section of Prescribing Information). Until these observations are better understood and clarified, however, and because renal adverse experiences have been reported with CLINORIL (see Adverse Reactions), caution should be exercised when administering the drug to patients with conditions associated with increased risk of the effects of nonsteroidal antiinflammatory drugs on renal function, such as those with renal or hepatic dysfunction, diabetes mellitus, complications associated with advanced age, extracellular volume depletion from any cause, congestive heart failure, sepsis, or concomitant use of any nephrotoxic drug. Discontinuation of NSAID therapy is typically followed by recovery to the pretreatment state. Since sulindac is eliminated primarily by the kidneys, patients with significantly impaired renal function should be closely monitored; a lower daily dosage should be anticipated to avoid excessive drug accumulation.
Use in Pregnancy - Not recommended for use in pregnant women, since safety for use has not been established and because of the known effect of drugs of this class on the human fetal cardiovascular system (closure of the ductus arteriosus) during the third trimester of pregnancy.

Nursing Mothers-Nursing should not be undertaken while a patient is on CLINORIL (Sulindac, MSD). It is not known whether sulindac is secreted in human milk; however, it is secreted in the milk of lactating rats.

Use in Children-Safety and effectiveness in children have not been established. Drug Interactions-DMSO should not be used with sulindac; concomitant administration has been reported to reduce plasma levels of active sulfide metabolite and potentially reduce efficacy and to cause peripheral neuropathy. Although sulindac and its sulfide metabolite are highly bound to protein, studies with daily doses of $400 \mathrm{mg}$ have shown no clinically significant interaction with oral anticoagulants or oral hypoglycemic agents; however, patients should be monitored carefully until it is certain that no change in their anticoagulant or hypoglycemic dosage is required. Special attention should be paid to patients taking higher doses than those recommended and to patients with renal impairment or other metabolic defects that might increase sulindac blood levels. Concomitant administration of aspirin significantly depressed the plasma levels of the active sulfide metabolite. Although addition of aspirin did not alter the types of clinical or laboratory adverse experiences, the combination showed an increase in the incidence of gastrointestinal adverse experiences; since addition of aspirin did not have a favorable therapeutic effect, the combination is not recommended. Concomitant administration of diflunisal in normal volunteers resulted in lowering of plasma levels of active sulindac sulfide metabolite by approximately one-third. Probenecid given concomitantly had only a slight effect on plasma sulfide levels, while plasma levels of sulindac and sulfone were increased; sulindac produced a modest reduction in the uricosuric action of probenecid, which probably is not significant under most circumstances. Neither propoxyphene hydrochloride nor acetaminophen had any effect on the plasma levels of sulindac or its sulfide metabolite.

Adverse Reactions: The following adverse reactions were reported in clinical trials or have been reported since the drug was marketed. The probability exists of a causal relationship between CLINORIL and these adverse reactions. The adverse reactions which have been observed in clinical trials encompass observations in 1,865 patients, including 232 observed for at least 48 weeks.

Incidence greater than 1\%: Gastrointestinal-Gastrointestinal pain (10\%), dyspepsia,* nausea* with or without vomiting, diarrhea * constipation * flatulence, anorexia, gastrointestinal cramps. Dermatologic-Rash,* pruritus. Central Nervous SystemDizziness,* headache,* nervousness. SpecialSenses-Tinnitus. Miscellaneous-Edema (see Precautions).

Incidence less than 1\%: Gastrointestinal-Gastritis, gastroenteritis, or colitis; peptic ulcer; gastrointestinal bleeding; Gl perforation; liver function abnormalities; jaundice, sometimes with fever; cholestasis; hepatitis; pancreatitis (see Precautions); ageusia; glossitis. Dermatologic-Stomatitis, sore or dry mucous membranes, alopecia, photosensitivity erythema multiforme, toxic epidermal necrolysis. Stevens-Johnson syndrome exfoliative dermatitis. Cardiovascular-Congestive heart failure, especially in patients with marginal cardiac function; palpitation; hypertension. Hematologic-Thrombocytopenia; ecchymosis; purpura; leukopenia; agranulocytosis; neutropenia; bone marrow depression, including aplastic anemia. hemolytic anemia; increased prothrombin time in patients on oral anticoagulants (see Precautions). Genitourinary-Urine discoloration; vaginal bleeding; hematuria; proteinuria; crystalluria; renal impairment, including renal failure; interstitial nephritis; nephrotic syndrome. Metabolic-Hyperkalemia. Psychiatric-Depression; psychic disturbances, including acute psychosis. Nervous System - Vertigo, insomnia, somnolence, paresthesia, convulsions, syncope, aseptic meningitis. Special Senses-Blurred vision, visual disturbances, decreased hearing, metallic or bitter taste. Respiratory-Epistaxis. Hypersensitivity Reactions-Anaphylaxis, angioneurotic edema, bronchial spasm, dyspnea, hypersensitivity vasculitis. A potentially fatal apparent hypersensitivity syndrome has been reported; this syndrome may include constitutional symptoms (fever, chills), cutaneous findings (rash or other dermatologic reactions-see above), involvement of major organs (changes in liver function, jaundice, pancreatitis, pneumonitis with or without pleural effusion, leukopenia, eosinophilia, disseminated intravascular coagulation, anemia, renal impairment, including renal failure), and other less specific findings (adenitis, arthralgia, myalgia, fatigue, malaise, hypotension, chest pain, tachycardia).

Causal relationship unknown: Other reactions have been reported in clinical trials or since the drug was marketed but occurred under circumstances where a causal relationship could not be established. However, in these rarely reported events, that possibility cannot be excluded. Therefore, these observations are listed to serve as alerting information to physicians. Cardiovascular-Arrhythmia. Metabolic-Hyperglycemia. Nervous System-Neuritis. Specia/ Senses-Disturbances of the retina and its vasculature. Miscellaneous-Gynecomastia.

Dosage and Administration: CLINORIL should be administered orally twice a day with food. The maximum dosage is $400 \mathrm{mg}$ per day; dosages above $400 \mathrm{mg}$ per day are not recommended. In osteoarthritis, rheumatoid arthritis, and ankylosing spondylitis, the recommended starting dosage is $150 \mathrm{mg}$ twice a day; the dosage may be lowered or raised depending on the response. A prompt response (within one week) can be expected in about one half of patients with osteoarthritis, ankylosing spondylitis, and rheumatoid arthritis: others may require longer to respond. In acute painful shoulder (acute subacromial bursitis/supraspinatus tendinitis) and acute gouty arthritis, the recommended dosage is $200 \mathrm{mg}$ twice a day; after a satisfactory response has been achieved, the dosage may be reduced according to the response. In acute painful shoulder, therapy for 7-14 days is usually adequate. In acute gouty arthritis, therapy for 7 days is usually adequate.

Management of Overdosage: Overdosage has been reported, and rarely deaths have occurred. In the event of overdosage, the stomach should be emptied by inducing vomiting or by gastric lavage, and the patient carefully observed and given symptomatic and supportive treatment. Animal studies show that absorption is decreased by the prompt administration of activated charcoal and excretion is enhanced by alkalinization of the urine

How Supplied: Tablets CLINORIL containing $150 \mathrm{mg}$ or $200 \mathrm{mg}$ sulindac, with cellulose, magnesium stearate, and starch as inactive ingredients, in unit-of-use bottles of 60 and 100 , unit-dose packages of 100 , and bottles of 100 .

Incidence between $3 \%$ and $9 \%$. (Those reactions occurring in less than $3 \%$ of patients are unmarked.

For more detailed information, consult your MSD Representative or see Prescribing Information. Merck Sharp \& Dohme, Division of Merck \& Co. INC., West Point, PA 19486 $\mathrm{JBCL} 72 \cdot 1(624)$ 
alone two. If something important warrants further explanation, as in this case, then the author should take time to shine light on the meaning rather than couch it in long words and run-ons.

That criticism aside, Dr. Kleinman exudes an integrity and honesty when writing about caring for people: "We must begin with the premise that chronic disease by definition cannot be cured, that indeed the quest for cure is a dangerous myth that serves patient and practitioner poorly. It distracts their attention from step-by-step behaviors that lessen suffering ... patient and practitioner both need to accept that the primary goal of treatment is the reduction of disablement in the ongoing experience of an illness." He continues, "The practitoner must relinquish the myth of cure."

The author includes many instances illustrating the art of the doctor-patientrelationship, particularly concerning what he calls the miniethnography. "Master ethnographers and clinicians," he states, "though their work is quite different, nonetheless tend to share a sensibility. They both believe in the primacy of experience."

The comparison is superb, both refreshing and dramatic. Dr. Kleinman uses many patients as models, such as Antigone Paget and Wolf Segal, to implement suggested improvements in their care. In comparing US and Chinese culture, he refers to Yen Guanghzhen, a patient with neurasthenia. For further cross-cultural descriptions, read Dr. Kleinman's $P a$ tients and healers in the context of culture.

Toward the end of the book in Chapter 15, Dr. Kleinman discusses his method of caring for the chronically ill. He writes, "The essence of that methodology is captured by the words empathic listening, translation, and interpretation, which I take to be the craft of the clinician who treats illness, not just disease ."

This methodology, with its emphasis on individual and cultural differences, serves as the key to the Kleinman philosophy. I hope we can learn more about his healthy and desperately needed insight in his future work. This book demands a certain academic fortitude, but more so the desire to improve as a person and physician. It is demanding, insightful, meditative, and at times displays a hard-won humor. Despite a few drawbacks, I highly recommend The illness narratives: Suffering, healing, and the human condition.

ROBERT A. NORMAN, DO

Arlington, Mass

\section{Maxillofacial trauma}

By Charles C. Alling III, and Donald B. Osbon. Pp 510, with illus. Lea \& Febiger, 600 Washington Sq, Philadelphia, 19106$9982,1988, \$ 80.00$.

According to the authors, the purpose of this book is to present detailed information concerning concepts, methods, and techniques of treating maxillofacial trauma. It is primarily targeted to residents within medicine and dentistry. Only those surgical techniques that are based on proven biotechnical applications, surgical anatomy, and physiology have been included.

Concisely written, the text covers a broad spectrum of current treatment modalities, with an emphasis on military injuries. General management and triage of the acutely injured patient; radiography and tomography; soft tissue injuries; and fixation prostheses, specifically methods and devices, comprise this section.

Separate chapters are devoted to the following areas: Mandibular and maxillary fractures; zygomatic arch and zygomatico-maxillary complex fractures; and, nasal-orbital-ethmoid and nasal fractures. Complex maxillofacial injuries, dentition and alveolar process injuries, and facial skeletal reconstructive surgery can be found here as well.

Diagrams and photos illustrate the techniques being performed. Similarly, before and after x-rays showing injury reduction, are well documented. Throughout the book, nicely delineated case studies prove useful. Maxillofacial trauma serves its intended audience well. It would make a fine addition to any hospital library.

JOHN ALTER, DO

Pontiac, Mich

\section{Books received}

\section{Health care}

Outpatient cancer centers: Implementation and management. Edited by Susan N. Nathanson and Dan Lerman; pp 269, with illus; American Hospital Publishing, Inc, 211 E Chicago Ave, Chicago, 60611, $1988, \$ 45.00$ (paper0, $\$ 35.00$ AHA members.

Privacy and confidentiality of health care information. By Jo Anne Czecowski Bruce; ed 2; pp 209, with illus; American Hospital Publishing, Inc, 211 E Chicago Ave, Chicago, 60611, 1988, $\$ 30.00$ (paper), AHA members $\$ 24.00$.

\section{Hematology}

Hematology for the house officer. By Larry Waterbury; ed 3; pp 155, with illus; Williams \& Wilkins, c/o Maple Press Fulfillment Center, York County Industrial Park M100, York, PA 17405, 1988, $\$ 14.95$ (paper).

\section{Internal medicine}

Internal medicine: Diagnosis \& therapy '88/'89. Edited by Jay H. Stein; pp 609, with illus; Appleton \& Lange, 25 Van Zant St, East Norwalk, CT 06855, 1988, $\$ 13.95$ (paper).

\section{Medical practice}

Essays on the rise and decline of bedside medicine. By Mark D. Altschule; pp 458, with illus; Lea \& Febiger, 600 Washington Sq, Philadelphia, 19106-4198, 1988, $\$ 29.50$.

The effective scutboy: The principles and practice of scut. By Robert A. Harrell and Gary S. Firestein; ed 3; pp 139, with illus; Appleton \& Lange, 25 Van Zant St, East Norwalk, CT 06855, 1988, $\$ 13.95$ (paper).

\section{Radiology}

Medicine's new vision. By Howard Sochurek; pp 179, with illus; The Radiological Society of North America, 1415 W 22nd St, Tower B, Oak Brook, IL, 60521, $1988, \$ 39.95$. 